\title{
LA ORGANIZACIÓN TERRITORIAL DEL ESTADO EN LA CONSTITUCIÓN DE 1991 ¿CENTRALISMO O AUTONOMÍA?
}

\author{
RICARDO ZULUAGA
}

\section{El debate constituyente}

Si bien la organización territorial del Estado es uno de los asuntos medulares de cualquier debate constituyente, la grave crisis de gobernabilidad que vivía el país a finales de la década de los 80, llevó a que cuando se inició el proceso de reforma de la Constitución de $1886,{ }^{1}$ este asunto no figurara entre las prioridades a afrontar, como sí lo eran los derechos fundamentales y sus garantías, así como ciertos órganos de la estructura estatal. Pese a ello, en los debates de la Asamblea Nacional Constituyente (ANC) de 1991 se presentaron, al igual que para casi todos los tópicos, numerosas propuestas de reforma, y que en este campo iban desde el mantenimiento del esquema entonces vigente, hasta una estructura federal. Sin embargo, la ANC no estuvo a la altura de su responsabilidad histórica y decidió, siguiendo un procedimiento inédito en el derecho comparado, no sólo no ocuparse del asunto, sino encomendárselo al legislador para que lo resolviera a través de una ley orgánica que hasta ahora no se ha expedido integralmente. En efecto, se han ido reglando unos aspectos aislados y fragmentarios, tales como la ley 60/93 (competencias), ley 128/94 (áreas metropolitanas), la ley 136/994 (régimen municipal), etc.; mientras que otras cuestiones fundamentales como el régimen departamental siguen en el limbo. Y ni qué decir de la creación de las provincias y las regiones, cuya entrada en vigor depende de la aprobación de dicha ley. Así pues, la definición de una materia que es por excelencia competencia del poder constituyente se convirtió en un asunto a ser resuelto por los poderes constituidos: legislador y juez constitucional, sin que ninguno de entre ambos haya cumplido a cabalidad su misión, como se verá.

\footnotetext{
${ }^{1}$ En estricto sentido debemos hablar de proceso de reconstitucionalización, pues nuestro proceso constituyente se dio realmente en la Constitución de Cúcuta de 1821; las demás, más que constituciones propiamente dichas, son reformas más o menos amplias a un mismo esquema. Sólo podría hablarse de una auténtica ruptura entre 1863 y 1886, lapso en el que la Constitución que rigió si significó una modificación profunda en el sistema imperante hasta entonces.
} 
Pese a esta realidad, desde hace una década, la doctrina jurídica nacional ha venido sosteniendo de forma insistente que la Carta Política aprobada en Colombia en julio de 1991 supuso un cambio radical y definitivo de la estructura constitucional que venía rigiendo en el país desde hacía más de un siglo. Pero una afirmación tan tajante sólo es parcialmente cierta, pues un análisis detenido de la Constitución nos permite evidenciar, con cierta facilidad, que el énfasis fundamental en los cambios operados se produjo en la llamada parte dogmática de la Constitución, aquella que recoge los principios y valores que orientan la acción del Estado, así como el catálogo de los derechos fundamentales y sus garantías. Mientras que la llamada parte orgánica, aquella que regula la forma y la intensidad del reparto del poder del estado, la que recoge las herramientas y mecanismos a través de los cuales se desarrolla el ejercicio del poder político, mereció una atención más bien secundaría en el curso de los debates de la ANC. ${ }^{2}$

\section{Los resultados: ¿Autonomía o descentralización?}

Si bien es posible afirmar con certeza que el énfasis realizado sobre la parte orgánica fue muy escaso, no es menos cierto que sobre esta materia si se introdujeron modificaciones, algunas de las cuales, como la Corte Constitucional, resultan notables. Pero en lo que atañe a la organización territorial del Estado, la Constitución de 1991 no introdujo cambios sustanciales, en la medida que el texto vigente sigue conservando el carácter unitario del Estado colombiano. Así se proclama tajantemente en el Preámbulo y en el art. $1^{\circ} \mathrm{CN}$ y ello supone desechar de plano cualquier asomo de organización federal.

Pero si en materia de organización territorial no es posible hablar de cambios radicales, tampoco se puede desconocer que se introdujeron una serie de modificaciones que nos permiten hablar de la existencia de elementos diferenciales entre la regulación de la Carta anterior y la de la actual. En primer lugar hay un cambio cuantitativo en el número de entidades territoriales, pues a las tradicionales (Departamento y Municipio) se han agregado las distintas clases de Distritos y los territorios indígenas y eventualmente, si el legislador así lo dispone, pueden llegar a operar las regiones y las provincias. Cualitativamente, esto es, en cuanto al grado e intensidad de las relaciones entre el centro y la periferia, es necesario desagregar el contenido del artículo $1^{\circ} \mathrm{CN}$, que define al Estado colombiano como unitario y de inmediato pasa a reconocer que es descentralizado y con autonomía de las entidades territoriales que lo integran.

En primer lugar, y como ya quedó dicho, cuando la Constitución en el preámbulo y en el artículo 1 define al Estado colombiano como una República unitaria, lo que hace es descartar la forma de estado compuesto -cuya manifestación más depurada es el tipo de estado federal- para asumir la forma de Estado simple. Esto implica que mientras no se produzca una modificación constitucional, las entidades territoriales

\footnotetext{
${ }^{2}$ Ahora bien, a favor de los miembros de ese cuerpo, es necesario decir que la especial atención que merecieron ciertos aspectos de la Constitución resulta entendible y aun justificable a la luz de las circunstancias en las que se dio el proceso constituyente que se desarrolló entre 1990 y 1991, pues para ese entonces, al igual que ocurre hoy, el país se veía sometido a unas condiciones de violencia y criminalidad que amenazaban con desintegrar al Estado.
} 
colombianas estarán en una relación de sujeción directa respecto del ordenamiento jurídico central, lo que dicho de otra manera, significa que no gozan de la posibilidad de decidir, mediante un documento originario llamado Constitución -manifestación de la voluntad soberana del pueblo- acerca de cierta forma de ser o sobre ciertas aspiraciones y ambiciones. No está de más advertir que en nuestro país el federalismo parece no gozar de amplias simpatías, pese a que ya ha transcurrido más de un siglo desde que la Reacción conservadora de 1886 diera al traste con la experiencia federal iniciada en 1853 y que había sido llevada a su máxima expresión con la Constitución de Rionegro de 1863.

En este mismo orden de ideas, cuando la Constitución reconoce el carácter descentralizado del Estado Unitario, realmente no está haciendo una particularización especial o introduciendo un elemento diferencial entre el ordenamiento precedente y el actual, en tanto la descentralización, como fenómeno administrativo, es propia de toda organización compleja. Ella, más que un reconocimiento jurídico, es una realidad que se impone en cualquier estructura organizacional, incluso en las de carácter privado y que con razón resulta más apremiante en las organizaciones estatales que no sólo deben asumir la gestión de múltiples y variadas competencias, sino que en algunos casos son de gran extensión territorial, tal como ocurre en Colombia.

El reconocimiento del carácter descentralizado del Estado colombiano supone excluir la centralización, que es una forma de Estado absolutamente imposible, para radicar en cabeza de las entidades territoriales el ejercicio de un determinado catálogo de competencias, que en todo caso estarán sujetas a unos controles de tutela por parte de la autoridad central. Desde ese punto de vista, las expresiones descentralización y autonomía resultan opuestas; sobre todo si se tiene en cuenta que etimológicamente autonomía significa independencia política y capacidad de regulación mediante normas y órganos de gobierno propios. ${ }^{3}$

Resta entonces dilucidar cuál es el alcance de la expresión autonomía, que es la que cierra la fórmula definitoria de nuestra organización territorial y que pareciera ser la más importante, de un lado porque como se anotó, el carácter descentralizado de nuestro Estado no es especialmente significativo y del otro, porque la Constitución no sólo la enuncia en el art. $1^{\circ}$, sino que la desarrolla y llena de contenido en su art. 287. Urge entonces, a los efectos que se persiguen con esta investigación, determinar el alcance o tipo de autonomía de que se habla en Colombia, sobre todo si se tiene en cuenta que desde el punto de vista jurídico es una expresión multívoca que comprende fenómenos muy disímiles y concretamente en materia de organización territorial, la expresión puede estar referida a manifestaciones diversas. Así, existe una autonomía política que faculta a los entes territoriales para realizar, a través de la ley, un desarrollo directo e inmediato de la Constitución, lo que les permite no sólo autoorganizarse, sino, sobre todo, encauzar sus aspiraciones regionales por la vía por ellos mismos determinada y sin mayores sujeciones al poder central. De la misma forma, existe una autonomía de carácter administrativo, según la cual la entidad territorial es la titular originaria de una serie de competencias administrativas que habrá de desarrollar de una forma más o menos independiente, sin imposición de directrices desde el sector central de la administración.

${ }^{3}$ Ello en los términos que trae el Diccionario de la Lengua Española de la Real Academia, $21^{a}$ edición. 
Clarificados los alcances de la noción de autonomía, resulta pertinente tratar de precisar a cuál de estos tipos es al que se refiere nuestra Constitución. En primer lugar, y de conformidad con lo que dispone el art. 150 de la Carta de 1991, la función legislativa está radicada de forma exclusiva y excluyente en el Congreso de la República, que es un órgano de carácter nacional. ${ }^{4}$ Luego, resulta claro que en Colombia las entidades territoriales no gozan de autonomía política. Lo anterior significa que la única autonomía posible es la administrativa, pero para precisar su alcance, es indispensable saber cuál es el contenido que la propia Constitución le ha dado a este concepto en el art. 287, que es donde lo caracteriza y desarrolla.

El análisis de estos elementos contenidos en el art. 287 se realizará individualmente, para determinar si ellos son una real manifestación de autonomía, aunque sea meramente administrativa, y para ello será necesario confrontarlos con otras disposiciones constitucionales y con los desarrollos legislativos y jurisprudenciales que se han llevado a cabo a lo largo de los diez años de vigencia de la Constitución.

\section{a. Gobernarse por autoridades propias}

El art. $287 \mathrm{CN}$ en su numeral $1^{\circ}$ dice que la autonomía territorial significa "gobernarse por autoridades propias", a lo que es necesario añadir que en los términos de los arts. 303 y $314 \mathrm{CN}$, las cabezas visibles de la administración local y seccional (alcaldes y gobernadores) son elegidos popularmente. Pero es la propia Constitución la que en diversos apartes atenúa el contenido de esta disposición. Así por ejemplo, en el inciso 4 de art. 115 adscribe a los alcaldes y los gobernadores a la rama ejecutiva, cuyo rector, como lo establece la misma disposición, es el Presidente de la República. De la misma forma, el art. 296, en materia de orden público, confiere una facultad preferencial al Presidente sobre los mandatarios seccionales y locales. Por su parte, el art. $303 \mathrm{CN}$ convierte a los gobernadores en agentes del Presidente en materia de orden público y de política económica general y los arts. 304 y 314.2 autorizan al Presidente para suspender o destituir a los gobernadores y alcaldes, lo cual resulta un procedimiento extraño, pues se permite que un funcionario que no guarda ningún tipo de relación jerárquica o funcional, pueda revocar un mandato que ha sido conferido directamente por el pueblo.

Y no menos es lo que ocurre con los órganos de representación popular seccionales y locales, cuya relevancia política es tan escasa, que es la propia Constitución la que en los arts. 299 y 312 convierte a las asambleas departamentales y a los concejos municipales en corporaciones exclusivamente administrativas y sin perder de vista que el art. $308 \mathrm{CN}$ permite que la ley establezca limitaciones a los montos que las asambleas pueden destinar para el pago de honorarios de los diputados y el funcionamiento de las contralorías

\footnotetext{
${ }^{4}$ En Colombia, el uso jurídico de la expresión Nación es muy problemático, puesto que se utiliza con un doble significado: en primer lugar con el tradicional, esto como comunidad que comparte una misma identidad cultural; pero históricamente también ha significado Estado central. Ambas acepciones son utilizadas indistintamente por la Constitución, así: con el significado sociológico (Preámbulo, arts. 2, 7, 63, 70, 72, $102,116,118,156,173,174,189.6,217,333$ y con el significado de persona jurídica: 9, 67, 128, 218, 266, 267, 268.3, 288, 298, 305.6, 305.11, 352, 356, 357, 359, 362, 364, 368. Esta enumeración es de Pedro Alfonso Hernández M: Descentralización, Desconcentración y Delegación en Colombia, Legis, 1999, págs. 8 y 9
} 
departamentales. Y algo similar ocurre con otras autoridades municipales, como el Personero, pues el art. $118 \mathrm{CN}$ más el art. 176 de la ley 136 y la ley 200, lo someten a la Procuraduría General, que es una autoridad nacional. Una situación parecida ocurre con las contralorías territoriales frente a la Contraloría General. En ambos casos, a pesar de no existir una dependencia orgánica, existe una dependencia funcional.

A la luz de lo expuesto, resulta fácilmente verificable que prescripciones de esta naturaleza, por el grado y la intensidad de las sujeciones a que someten a las autoridades locales y seccionales respecto de las centrales, en la práctica desfiguran significativamente la autonomía de las autoridades territoriales, a tal punto, que más parecieran ser simples agentes del gobierno central. Sobre todo si se tiene en cuenta que la autonomía no se reduce a la mera escogencia popular de los mandatarios, tal como ocurre en Colombia, sino que ella demanda que los mismos estén revestidos de facultades y poderes autónomos.

No obstante todas las sujeciones descritas, la Constitución de 1991 introdujo algunos cambios positivos son: la elección popular de gobernadores y la unificación de la totalidad de las entidades territoriales seccionales, pues antes había inmensos territorios (intendencias y comisarías que eran administrados directamente por el ejecutivo nacional a través del desaparecido DAINCO, un simple departamento administrativo dependiente directamente del Presidente de la República).

\section{b. Ejercer las competencias que les corresponden}

De la misma forma, cuando el art. $287 \mathrm{CN}$ dice en el numeral $2^{\circ}$ que es una manifestación de autonomía de las Entidades Territoriales "ejercer las competencias que les correspondan", es necesario tener en cuenta otros enunciados constitucionales para precisar cuál es el alcance de esta expresión. De esta manera, los arts. 150 y 189 de la Constitución establecen un largo y exhaustivo listado de 53 materias cuyo desarrollo, a cargo del Congreso y del Presidente, prácticamente agotan los cometidos estatales esenciales. Mientras que el catálogo de materias que la Constitución asigna a los departamentos y municipios en los arts. 300, 305, 313 y 314 carecen de alcance real o cuando lo tienen, están supeditadas a lo que disponga el legislador, que es un órgano nacional, con lo cual las competencias originarias y aptas para que las entidades territoriales generen un impacto político, social y económico real, son prácticamente inexistentes. A ello se añade que en los términos del art. 189 numeral 11, el monopolio de la potestad reglamentaria de las leyes está radicado en el Presidente, con lo cual las competencias que corresponden a las entidades territoriales no sólo estarán intermediadas por la ley, sino también por lo que en cada caso disponga el jefe del ejecutivo nacional. De esta forma, las competencias de los entes territoriales dejan de ser propias y se convierten en meras competencias de ejecución y desarrollo de la voluntad del gobierno central.

Un caso que ejemplifica perfectamente esta situación es el de la prestación de los servicios públicos que, de acuerdo a los arts. 311 y $367 \mathrm{CN}$, está considerada como una de las funciones municipales por excelencia. Pues bien, la propia Constitución le encomienda al Presidente de la República "ejercer la inspección y vigilancia en la prestación de los servicios públicos" (art. 189, numeral 22), a la vez que redobla esta facultad en el art. 370, que dice que "corresponde al Presidente de la República señalar, con sujeción a la ley, las políticas generales de administración y control de 
eficiencia de los servicios públicos domiciliarios y ejercer por medio de la Superintendencia de Servicios Públicos Domiciliarios, el control, la inspección y vigilancia de las entidades que los presten". Igual suerte corre la acción educativa, cuya gestión se confía de forma conjunta a la nación, los departamentos y municipios (arts. 67 inciso 6 y 300 numeral 10 $\mathrm{CN}$ ), pero la facultad compartida se reduce notoriamente, pues la propia Constitución encomienda al Presidente de la República en el art. 189 numeral 21: "Ejercer la inspección y vigilancia de la enseñanza conforme a la ley".

Pero las sujeciones no sólo se establecen respecto del sector central de la administración, sino desde lo seccional hacia lo local. Así, en los términos del art. 305.10 CN, los gobernadores tienen la facultad para revisar los actos de los concejos y los alcaldes y si los consideran inconstitucionales o ilegales, los remiten al Tribunal Contencioso Administrativo para que resuelva sobre su validez. Y el art. 314.2 faculta a los gobernadores para suspender o destituir a los alcaldes.

Además de lo anterior y a pesar de que los repartos de competencias son tan estrictos y de que el sector central se reserva la dirección funcional y operativa de la mayor parte de ellas, de forma solapada traslada a las entidades territoriales, especialmente a los municipios, los costos financieros que genera el funcionamiento de muchas de las competencias que le corresponden y así los municipios terminan sufragando una porción significativa de las funciones que son responsabilidad del sector central de la administración, como la seguridad pública, la justicia y la función electoral, que de no ser subsidiadas por estos, se verían completamente paralizadas.

c. Administrar los recursos y establecer los tributos necesarios para el cumplimiento de sus funciones

Igual suerte corre el tercer elemento constitutivo de la noción de autonomía y que consiste en la facultad de las entidades territoriales de "Administrar los recursos y establecer los tributos necesarios para el cumplimiento de sus funciones". Se trata de un elemento de vital importancia, pues en última instancia, la verdadera autonomía, más allá de la asignación de competencias, radica en la disponibilidad de los recursos necesarios que le permitan a las entidades territoriales materializar los cometidos concretos que les corresponde desarrollar, a la vez que son los ingresos monetarios los que aseguran que la entidad territorial subsista autónomamente. Y si bien es verdad que existe un buen número de impuestos territoriales, los mismos, de conformidad con lo establecido en los arts., 300.4 y $313.4 \mathrm{CN}$, solamente se pueden crear bajo las condiciones que establezca el legislador nacional, que es también el que determinará los parámetros a partir de los cuales se pueden recaudar los demás ingresos básicos de los entes territoriales (operaciones de crédito interno y externo, operaciones de mercado y tasas y tarifas por la prestación de servicios públicos).

En estas condiciones es difícil, cuando no imposible, hablar de autonomía fiscal; sobre todo si se tiene en cuenta que ella supone tanto disfrutar de la capacidad de generación, como la de disposición de los bienes y recursos. A ello hay que agregar que el estado central está provisto de otra serie de instrumentos como la ley general de presupuesto, el plan general de desarrollo, el sistema de contabilidad nacional, la legislación sobre control fiscal, entre otros, que le permiten intervenir sobre las finanzas 
locales y seccionales para dirigirlas, no sólo en el monto y en la forma del gasto, sino indicando las áreas en las que estos recursos deben ser invertidos. De esta manera, los recursos provenientes de las transferencias (situado fiscal e ICN), están rígidamente dirigidos, así: educación $30 \%$, salud $25 \%$, agua potable y saneamiento básico $20 \%$, educación física $5 \%$ y el $20 \%$ restante, que quedaba para libre inversión, ha sido destinado por los Conpes a electrificación, equipamiento municipal, desarrollo comunitario, pago de deuda por inversiones en estos tres sectores y en vías. Mientras que los recursos que se pueden obtener a través de fondos de cofinanciación tienen que obedecer a proyectos evaluados técnica, económica y financieramente por la Nación y que demandan siempre una contrapartida. De la misma forma, las regalías que reciben los departamentos y los municipios productores y los municipios portuarios, son recursos que están destinados en su totalidad a inversión en proyectos de progreso municipal contenidos en el plan de desarrollo.

\section{d. Participar en las rentas nacionales}

Por último, el art. 287 dice que "participar en las rentas nacionales" es una manifestación de autonomía, cuando en realidad se trata de un enunciado cuyo alcance no parece tan claro, pues él supone establecer un nexo de dependencia entre el centro y la periferia, donde a ésta última lo que le queda es la posibilidad de acceder al disfrute de unos recursos que son titularidad de la Nación. Además, las rentas nacionales que se trasladan a los entes territoriales (transferencias, regalías y fondos de cofinanciación), como se vio, son recursos que vienen con una destinación específica en sectores y porcentajes y cuya inversión es supervisada desde el sector central de la administración. Aparece claro que de lo que se trata es de una mera gestión de recursos, cuando lo que resultaría una verdadera manifestación de autonomía es permitir que las entidades territoriales puedan generar los recursos necesarios que demande su funcionamiento. Reconocer el derecho a participar en estas rentas, no es otra cosa que establecer un lazo de subordinación entre la administración central, que recauda y distribuye el grueso de la carga tributaria del país y las administraciones territoriales, que se deben limitar a esperar que desde el centro se les transfieran esos recursos, a veces más como una graciosa concesión, que como un derecho.

\section{La estructura territorial resultante}

Si cualitativamente la situación parece poco alentadora, cuantitativamente el panorama no es menos oscuro. En primer lugar, la Constitución ha diseñado un modelo territorial caótico, pues promueve la competencia entre alternativas que son excluyentes entre sí: región, departamento y provincia, cuando el derecho comparado ha demostrado de forma suficiente la inviabilidad de este tipo de regulaciones. Así por ejemplo, en España, la provincia, que es la entidad territorial que se interpone entre la Comunidad Autónoma y el municipio, actualmente está sumida en una grave crisis que la tiene al borde de la desaparición. Pero nuestro modelo constitucional adolece también de una gran inflexibilidad, pues las entidades territoriales posibles (regiones y provincias) han sido previstas como 
una camisa de fuerza, ya que para su formación deben partir de la base de las entidades territoriales existentes y no de realidades históricas, económicas y sociales, lo cual es una lástima, porque en Colombia todavía sigue siendo relativamente fácil elaborar un mapa territorial, dada la inexistencia de fuertes tensiones regionales. Finalmente, es necesario decir que la división territorial colombiana no obedece a ningún principio racional. Así, los departamentos han quedado prácticamente desmantelados, pues la casi totalidad de las funciones y cometidos estatales se le encomiendan a la Nación y a los municipios. Además el art. 311 introduce un elemento distractor al decir que el municipio es la entidad fundamental de la división político-administrativa del Estado a partir de lo cual es necesario preguntar si nuestra Constitución proyecta un Estado fundado en una autonomía de carácter municipal o en una de carácter seccional.

\section{Colofón}

Un cuadro como este arroja un balance bastante negativo para la noción de autonomía, incluso si es la administrativa; panorama que resulta más desolador si se tiene en cuenta que la mayor parte de estas sujeciones vienen determinadas por la propia Constitución, con lo cual, un cambio sustancial de la actual realidad territorial debe pasar necesariamente por el dispendioso trámite de la reforma constitucional. A lo que se añade que una situación como la presente, ha venido a agravarse por actitudes tanto del legislador como de la Corte Constitucional, pues ellos, primeros llamados al desarrollo de la Constitución, han privilegiado una lectura profundamente centralista de la Carta de 1991. Prueba fiel de ello son muchas de las leyes territoriales expedidas hasta ahora, desde la 136/93, que reguló incluso los detalles más insignificantes de la vida municipal, hasta la 617/00, que con el eufemístico encabezado de "Instrumentos para profundizar en la descentralización", entra a saco en los escasos y exiguos márgenes de autonomía de que disponían los municipios, para ordenarles recortes de gasto, supresión de dependencias y en general, imponerles unos rígidos y severos programas de ajuste fiscal, que son una prueba más de un incisivo proceso recentralizador, mediante el cual el Estado central está coartando la libertad de acción de las entidades periféricas, aduciendo su corrupción e ineficacia, pero sin demostrar que él no incurre en las prácticas y vicios que con tanta severidad reprime. ${ }^{5}$

Nuestra realidad territorial pudo y debió correr una mejor suerte si los principios rectores que inspiraron el nuevo texto constitucional hubiesen sido objeto de un desarrollo coherente en su parte orgánica. Así se hubiera evitado la carencia de contenido que hoy caracteriza a ciertas partes del texto y que se facilita, como ha dicho Sandra Morelli “... porque paradójicamente, se reconoce una contradicción axiológica entre la parte dogmática y la parte orgánica de la Constitución, en lo que concierne al régimen territorial, contradicción que se ha interpretado por el legislador y por el juez constitucional, en beneficio de la centralización, de tal manera que la autonomía es letra muerta en nuestra realidad institucional y el fantasma de la centralización política y descentralización administrativa no sólo sigue rondando por los corredores del Capitolio, del Palacio de Nariño y, en la

${ }^{5}$ Cfr. Hernández M., Pedro Alfonso: ibídem, pág. 69 
calle 72 donde funciona la Corte Constitucional, sino que parece impregnar todas las instancias decisorias del Estado e incluso del inconsciente colectivo". ${ }^{6}$

Muchas de estas contradicciones se podrían solventar y orientar en un sentido diferente a través de la hasta ahora frustrada Ley Orgánica de Ordenamiento Territorial, cuya expedición integral no ha sido posible en el curso de los diez años de vigencia de la Constitución. Como ya se dijo, solamente han aparecido menciones desarticuladas que se han ocupado de la organización territorial en una multiplicidad de normas como las leyes 60, 128, 136 y 617. Probablemente para cuando el legislador decida afrontar con rigor y seriedad el problema del reparto del poder entre el centro y la periferia, las políticas neoliberales, aupadas por la corrupción política y la ineficacia pública, hayan obligado al desmantelamiento del aparato estatal para entregarlo a los particulares. Ese día entonces, no habrá problema territorial que resolver. 